\title{
AttendanceBot Location based Attendance Tracker for Workers
}

\author{
Bryan Nii Lartey Laryea \\ Business and Information Service \\ Department \\ CSIR-Institute of Industrial Research, \\ Ghana \\ William O. M. Addo
Business and Information Service
Department \\ William O. M. Addo
Business and Information Service
Department \\ William O. M. Addo
Business and Information Service
Department \\ Ghana \\ Kennedy Andoh \\ Business and Information Service \\ Department
}

\begin{abstract}
Employees are expected to report on time for work and be productive. In Ghana, it is mandatory for governmental departments and agencies to record attendance of employees to work using biometric technologies to prevent attendance fraud. However, some infrastructural decisions can hinder staff not to register their attendance on time such as the devices to scan their features are quite far from the entrance to these agencies. It is not unusual for workers to forget to record their attendance. In the paper the experiment was built on existing technologies to prevent duplication of effort as much as possible hence the opportunities of smartphone ubiquity and its features were exploited to mitigate the stated problems. A bot was built riding on the Telegram messaging platform to implement the design. All programming for this experiment was done in Java. Springboot Framework was used to get this work done. Data captured was stored in Redis.
\end{abstract}

\section{General Terms}

Locational based Attendance tracking, Telegram Bot Authentication Algorithm

\section{Keywords}

Bot, Attendance Authentication, Telegram, Geo-Fence

\section{INTRODUCTION}

Employers are much interested in productivity of staff as it correlates to profitability and the growth of the company. One way staff productivity is measured is the amount of time they spend on the job or the man-hours by attendance management. Attendance management is the act of managing attendance or presence in a work setting, which maximizes and motivates employee attendance thereby minimizing loss. Not only does it affect productivity, it can reduce the company profits or even additional contracts [1]. Every firm has a reporting time and closing time. The difference between the two is the time or the man-hours of the staff spent at work. Employers loathe paying staff more than what they work for and hence there is the need to record attendance. The first recording was done with the punch card and attendance recording books. This is effective when the workers are honest. However, human behavior is unpredictable; hence we do not leave much room for speculation. Modern attendance management systems use the biometric system to record staff attendance to mitigate the shortfalls of the manual recording as no two persons share the same biometric features. Biometric technologies generally refer to the use of technology to identify a person based on some aspect of their anatomy [2]. This can be iris detection or fingerprint or both. This works well than the manual recording as staff data can be computed very quickly due to data storage in embedded database in the devices or external data storage which can be queried as compared to the manual tabulating of attendance records. Offices that have the biometric attendance registers often have the biometric attendance devices placed at a central location where staff have to walk to capture their biometrics information to record their attendance. This is sometimes done to prevent theft and tampering of the devices or closer to the nearest power supply. These devices are expensive and the takes time to install and enroll staff fingers.

The location of the devices can be a factor in recording a person late. Some devices are quite far even as much as $500 \mathrm{~m}$ from the entrance or boundaries of the office premise. Average walk speed is $1.4 \mathrm{~m} / \mathrm{s}$ [3] hence distance of the biometric device can result in employees being recorded late even when they get to work on time. So much happens during journey to the office such as traffic, unreliable transportation system etc. hence time is precious and every second should be saved in order to report to work early. These problems have to be solved creatively. Smart phones are common now and most workers in the public sector have access to smart phones. Most smartphones have a Global Positioning System (GPS) sensors embedded in them. The GPS helps in getting the location of the users. The location position received from the GPS can also be used to get directions to a chosen destination from one's present location. The GPS in smart phones can be used to capture attendance using their location to the office [4]. Employees do not need to walk to the biometric device when this is implemented. They only have to record their attendance using the smartphone. There are many studies and experiments using this technique. These techniques mainly demand that the office premise have a Geo-Fence or a virtual fence using the GPS coordinates of the boundaries of the office complex. A Geo-Fence is a virtual perimeter for a realworld geographic area. A Geo-Fence could be dynamically generated-as in a radius around a point location, or a GeoFence can be a predefined set of boundaries. This technique has been used in advertising where mobile users are targeted within a predefined virtual geographic boundary around an advertiser's establishment [5]. We used this concept to design our system in a way that when the staff send their location within the Geo-Fence in relation to their GPS coordinates their attendance are recorded otherwise it is rejected. Usually this system is deployed to mobile staff when they go assignment on the field. Many developers have developed mobile applications

that does this. The aim of this paper is to implement this with less programming to save time and money by using freely available technologies. In this paper we leverage the power of bots to record attendance and also remind staff who have not signed in. Bots are now in vogue. Many companies use bots for customer interactions. In their work, Polluti-Bot: An informative bot of pollution in Madrid, Julio Manuel GarcíaMartín used Telegram Conversational Bot to inform users of the level of pollution in Madrid, showing the current level of 
contamination in Madrid and make automatic pollution notifications based on the vehicle's eco-label and information on parking occupancy. Telegram bot framework was selected for this work. Telegram bot framework is very easy to use and expose all the features of the Telegram messaging application. Another reason is that there are versions of the messaging application on for Windows OS, Linux, iOS and Android. Therefore, there is no need to code for individual platforms. The API for the bot framework is available for many programming languages. Telegram message application has the feature to send location to contacts. Using this feature users can send locations to our attendance bot which would be analyzed to check when the staff is in the virtual fence. All the implementation would be done in Java. In this experiment Redis, Java, Spring boot, Thymeleaf and HTML were used. The experiment was conducted at the CSIR Industrial Research Institute's campus. At the Institute, the biometric attendance device is located approximately $200 \mathrm{~m}$ from the main entrance and $150 \mathrm{~m}$ from the side entrance. The reporting time to work is 8:30 am. Sometimes the location of the device has a queue of staff wanting to register their reporting time. A staff averagely spends 5 seconds to register the time they report to work. However, there are instances staff have to spend more time because the device cannot recognize them. This could happen either because they are not placing their thumbs well on the finger print scanners or don't have clean thumbs. This can result in lateness of employees stuck in the queue.

\section{Table. 1 Walking speed by ages}

\begin{tabular}{|l|l|}
\hline \multicolumn{1}{|c|}{ Age } & \multicolumn{1}{|c|}{ Meters/second } \\
\hline 20 to 29 & 1.34 to 1.36 \\
\hline 30 to 39 & 1.34 to 1.43 \\
\hline 40 to 49 & 1.39 to 1.43 \\
\hline 50 to 59 & 1.31 to 1.43 \\
\hline 60 to 69 & 1.24 to 1.34 \\
\hline
\end{tabular}

\section{RELATED STUDIES}

Several studies have been conducted in the field of attendance tracking using different technologies and strategies. Ankur Chandra et al in their paper GPS Locator: An Application for Location Tracking and Sharing Using GPS for Java Enabled Handhelds; gives a detail on their then ongoing project work in the field of Location Based Services for JAVA enabled mobile devices, equipped with GPS receptor. They presented a novel technique to send GPS coordinates to other mobiles through Short Message Service (SMS) based on Global Positioning System (GPS) technology. This application also enabled users to get their current location coordinates (latitude, longitude and altitude) and they can also view their locations on the Google maps. Further, their application also enables the user to share his location with their friends through a web server using internet connectivity in their handhelds.

Shermin Sultana et. al [8] proposed a smart, location-based time and attendance tracking system using Android application. This works when the employee enters the office area. The android device of the employee is automatically connected to the office internet and a message is sent to the office sever with the employee id and local time of the device which is counted as login time of that employee. When employee leaves the office area, a message is sent to the office server with employee id and local time which is counted as logout time. The limitation of their system is that, it is limited to android operating system. It needs android location service to be running always which can drain the battery of the device. It takes time to deploy as separate applications have to be developed for every mobile operating system from ground up. In case the wireless network of the company is off or out of range because of wireless signal blockers like trees, employees cannot login.

M.A. Ansari et. al ,in their paper Event Organization Using GPS Based Location Tracking Including Communication System, they experimented the use of smartphone GPS sensor to allow other locations to be plot on a map for others to see by permission and it is ideal for event organization. Their system also has short message feature that can be used for inter user communication. However, their model does not include a Geo-Fence which would serve as boundary to discard unwanted location if their solution would be used at a work place. They also used android application which would disenfranchise some users. Using Short Messaging Service (SMS) is expensive as compared to using smartphone messaging applications like Telegram to send messages and images.

In their paper Machine Learning Biometric Attendance System using Fingerprint Fuzzy Vault Scheme Algorithm and Multi-Task Convolution Neural Network Face Recognition Algorithm, 2018 Patrick Cerna et al. experimented fingerprint and face recognition to track staff attendance to work using low energy consuming devices like Raspberry Pi and Arduino with their complementary sensors to demonstrate this. Their concept mandates the staff to walk to where the devices are, to record their presence at work.

To solve the limitation of having a wired attendance system Jianpo $\mathrm{Li}$ et al proposed a design method of wireless fingerprint attendance system based on ZigBee technology they realized low-cost and high-performance wireless fingerprint attendance function, which provided a new wireless fingerprint attendance system for enterprises and institutions. However, this required having additional hardware which relied on ZigBee technology and setting up needs much the technical know-how.

Stephen A. Coetzee and Astrid Schmulian at Department of Accounting, University of Pretoria, South Africa demonstrated how they used a messenger bot to track student attendance to provide data on student attendance to tackle poor performance [13]. Their method did not enforce a Geofence and check location spoofing.

\section{METHODOLOGY}

The experiment was done in two phases. The first phase is the setting up of the bot and the user interaction with the system and the second phase is design of a web interface to view data of attendance record. Before proceeding the features of the bots should be identified. The following features was settled on.
a. register with staff id
b. bot prompts for full name
c. bot prompts for picture of staff.
d. User enters the location. 


\section{First Phase}

\section{Bot Registration}

The requirement of Telegram bot is the registration of the bot. This is done via the Telegram's BotFather bot in the Telegram messenger application. This requires the user to choose a bot user name and bot name following a strict naming convention. A unique token is generated following a successful registration. Fig. 1 shows the bot registration using bot father.

\section{Geo-Fence Creation}

A Geo-Fence of the campus of the Institute of Industrial Research was created by taking the coordinates of the four corner edges of the walls. A smartphone GPS was used to take the coordinates. The smartphone was selected instead of a dedicated GPS device in order to get the coordinates with the accuracy level of the smartphone as that would be used by users in real-world application. In the Spring Boot application d a Geo-Fence was created using polygon object using found in the Spatial4j Library. Any coordinate would be made a Point object to check if it is within the polygon. Fig 2. is the code that checks if the location position in the GeoFence polygon

\section{Telegram Bot Programming}

Java was the main programming language in this experiment hence the Java library for the Telegram Bot API was used. This experiment would be implemented using Spring Boot framework hence the Telegram Spring Boot Starter Telegram Bot Java Library was chosen [9]. The Spring Framework is an application framework and inversion of control container for the Java platform. The framework's core features can be used by any Java application, but there are extensions for building web applications on top of the Java EE platform. Redis serves as the datastore for attendance records of the staff. Redis is an open source (BSD licensed), in-memory data structure store, used as a database, cache and message broker [12]. Reading and writing to the Redis data store is faster than the RDMS like MySQL as there is no disk write and read. The concurrent access to Redis is very high. The default connection is 100000 concurrent hits.

\section{Staff Registration by Bot}

The employee has to append their staff number when they type /register command i.e. /register 109049. Telegram generates a unique ID token to every user. This token which is a long value does not change. Once entered our bot receives it and creates a user schema in the Redis data store with the generated unique number named as chatid. The staff number and the chatid data is stored in Redis hash in the pattern staff:staff_number and a list name as ids which contains the auto generated chatid. The list would be used to iterate the hashes to get all staff information for retrieval. A successful registration prompts the user to enter their name. This is appended to the hash created in the first registration step. A successful update to the user hash map prompts the user to upload a picture to complete the registration. The image taken is stored on Telegram's servers and they are given a unique key known as fileid. The generated fileid was sent to the Redis server for storage and later retrieval

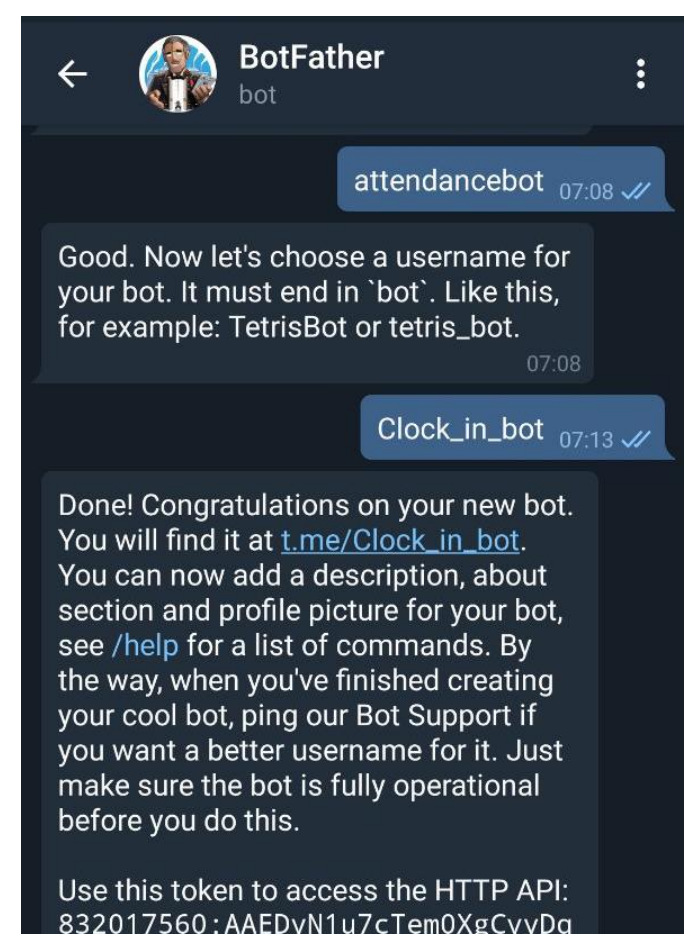

Fig 1: This captures a successful bot creation and registration.

public static boolean onCampus(Location location) \{

Coordinate $\mathrm{p}=$ new Coordinate (location.getLatitude $($ ), location.getLongitude());

GeometryFactory geometryFactory = new

GeometryFactory();

Point point $=$ geometryFactory.createPoint $(\mathrm{p})$;

Coordinate[] coords $=$ new Coordinate[ $]\{$ new

Coordinate(5.658978, -0.151075),new Coordinate(5.659181, -

0.147486), new Coordinate(5.655577, -0.147316$)$,new

Coordinate(5.655491, -0.149958), new Coordinate(5.658978, $-0.151075)\}$

LinearRing ring $=$

geometryFactory.createLinearRing(coords);

LinearRing holes[] = null;

Polygon polygon $=$ geometryFactory $\cdot$ createPolygon $($ ring, holes);

return point.contains(point);

\}

Fig 2: Code to create a Geo-Fence polygon object and check if a location is within the polygon

Attendance Data Capture

The java Telegram bot API supports Location messaging. The Location class has methods to get latitude and longitude from the Location Messages. The two values would be used to get if the point exists in the Geo-Fence when the user sends it to the bot. Testing the sending of location revealed a flaw. Users can forward location messages to the bot which would be treated as valid entry. To eliminate this flaw an algorithm was formulated as a solution.

1. if message arrived has locations.

2. Check for forwarded sender id 
3. if id is null then check if the position is in the polygon of the Geo-Fence.

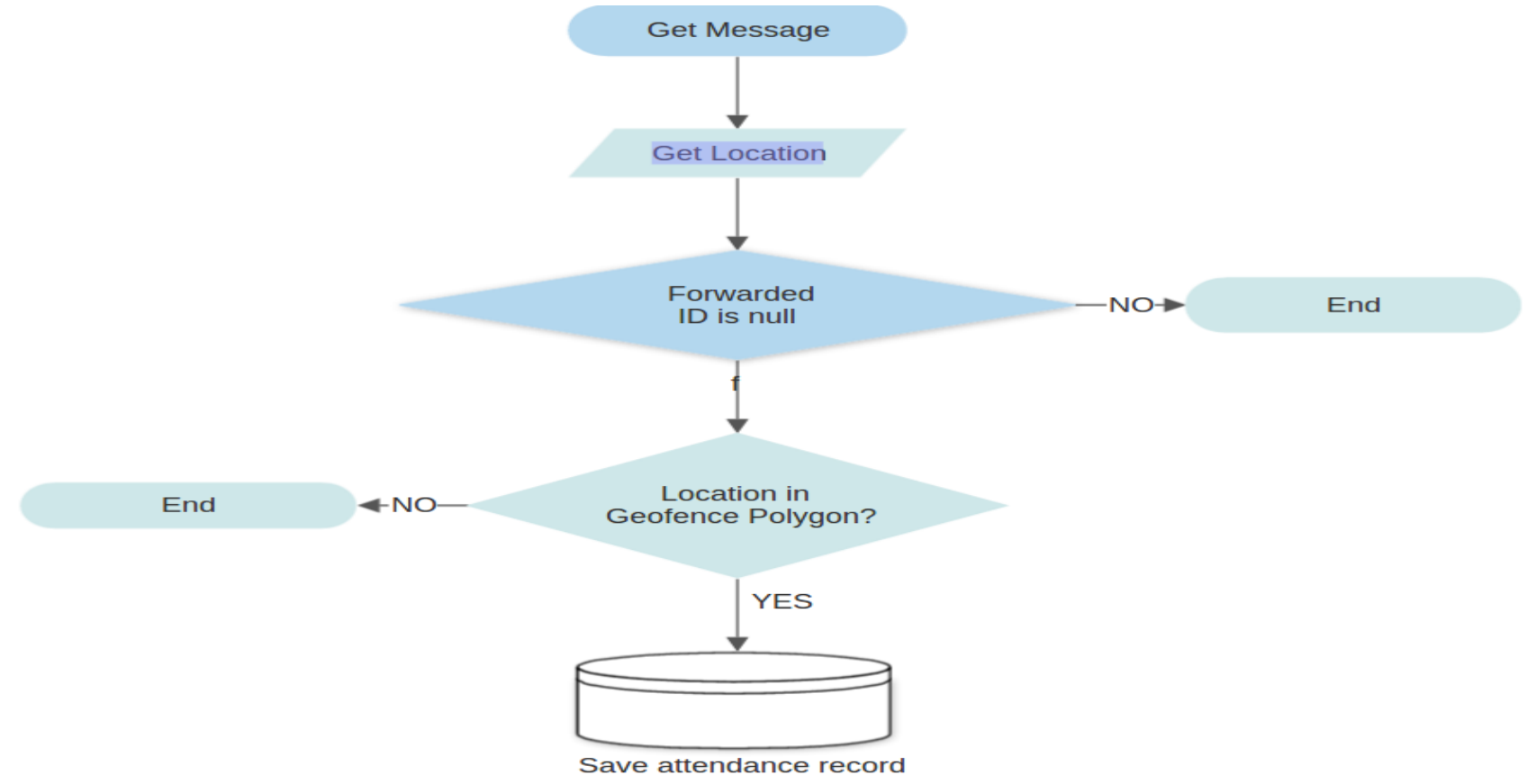

Fig 3: The process flow.

\section{Attendance Reminder}

It is not unusual for staff to forget to register their attendance to work. This can be due to forgetfulness, work related stress or personal problems. Giving excuses for not registering your attendance is not acceptable even though some have good reasons. Leveraging the push notification of Telegram bot HTTP API staff can be alerted when they fail to register their attendance by a certain time. The http request was used for this using the following URL structure in our code https://api.Telegram.org/bot[BOT_API_KEY]/sendMessage?c $h a t \_i d=\left[M Y \_C H A N N E L \_N A M E\right] \& t e x t=\left[M E S S A G E \_T E X T\right]$. At Institute of Industrial Research, the arrival time is 8:30 am so our bot sends out the alert to staff with no attendance data on that day at 8:25 am. We added extra features to prevent the bot sending messages to the staff on holidays. The holidays of the year data were scrapped from https://publicholidays.africa/ghana/2019-. In Ghana when the holiday is on a weekend, the following week day is a holiday. Also, the bot does not send reminders on weekends. These rules were factored in this experiment. We created a set for each new date. This set contains the chatids which can be matched to a staff. When it is time for the reminder to send messages, the difference between the ids and attendance data sets is computed. The resulting set of members are workers who are yet to record their attendance. This set of ids are looped to send messages. To keep the reminder up and running, the Springboot Scheduler was activated. The scheduler runs the task using the cron parameter we set in the code.

\section{Attendance Data Processing}

This step involves structuring the data received in a format before storing them. The team chose Redis hash data structure to store our attendance data. Every staff has a hash list with the label attendance:chatid where the chatid is the unique user generated number by Telegram and the date as the key with the time reported to work as the value as shown in Fig.6. The put function was called to store the data in the hash. The team chose to use hash to store attendance data to

make it possible to check the time the worker reported to work instead of writing a loop to check for it in the case we used a loop. The time reported to work was converted to minutes to easily know the staff is late, on time or early. Staff reporting time 8:30am is recorded as integer of 510. When the converted number representing the time of attendance is less than 510 then worker is early but when more than 510 , worker is late.

if (message.hasLocation()) \{Location

localarg0.getMessage().getLocation(); try \{

long $\mathrm{t}=\arg 0 \cdot \operatorname{getMessage}() \cdot \operatorname{getForwardFrom}() \cdot \operatorname{getId}() ; \mid$

SendMessage message $=$ new SendMessage (chatid, "Can not accept forwarded locations");

execute(message);

\}

Fig 4: Checking for location spoofing by forwarding already sent location data code snippet

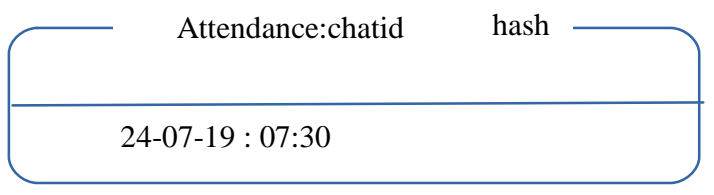

Fig 6: Redis list for attendance 


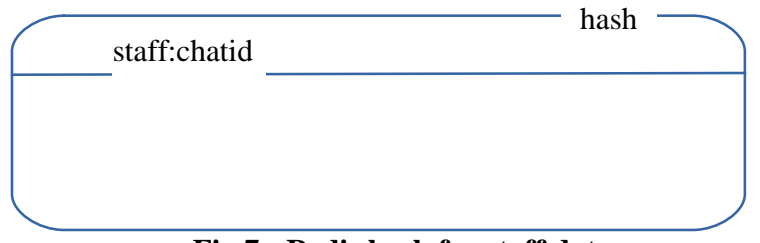

Fig 7: Redis hash for staff data

\begin{tabular}{|ll}
\hline 132321321 & set \\
\hline $2019-07-21$ & \\
\hline
\end{tabular}

Fig 8: Redis set for dates recorded staff

ids set

132321321

Fig 9: Redis list for staff ids

\section{Second Phase}

\section{Data Presentation}

The experiment would not be complete without a data visualization layer. This would let users know whether the attendance data was captured well as well as the algorithm. Springboot MVC to was used to create the web interface with the help of Thymeleaf template engine. Thymeleaf is a modern server-side Java template engine for both web and standalone environments. Thymeleaf's main goal is to bring elegant natural templates to your development work flow With Thymeleaf HTML that can be correctly displayed in browsers and also work as static prototypes, allowing for stronger collaboration in development teams.

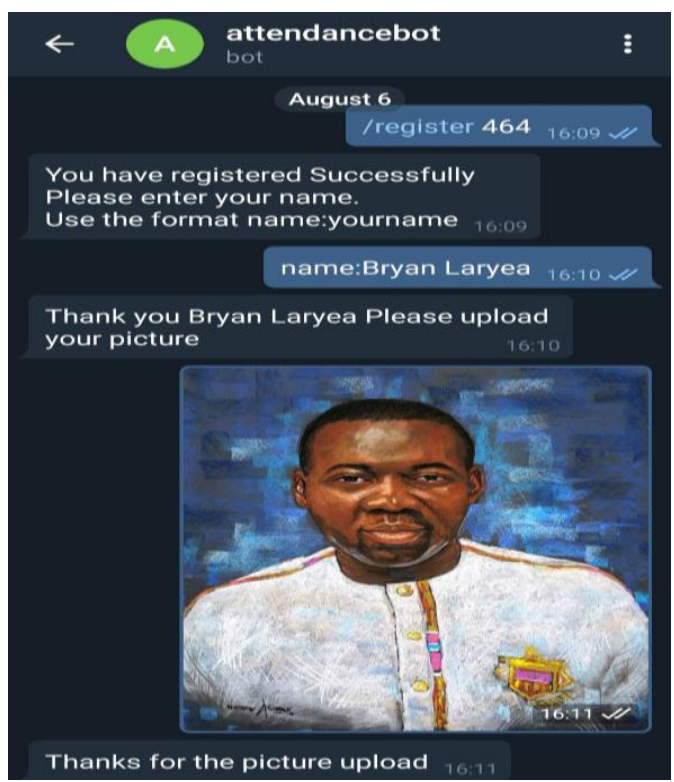

Fig 10: Successful registration on Android OS.

\section{EXPERIMENT RESULTS}

The experiment was tested by five selected staff. They installed Telegram messaging application on their mobile phones. They login to the application every morning for 10 days. The data visualization in the web application is done by Spring-boot MVC. Fig 10 shows the registration process for staff using bot on Telegram messages mobile application. A successful operation allows user to proceed to the next stage.

Fig 11 shows the recording of attendance of staff. After a successful location position upload, the chat bot returns with a confirmation and status of your attendance

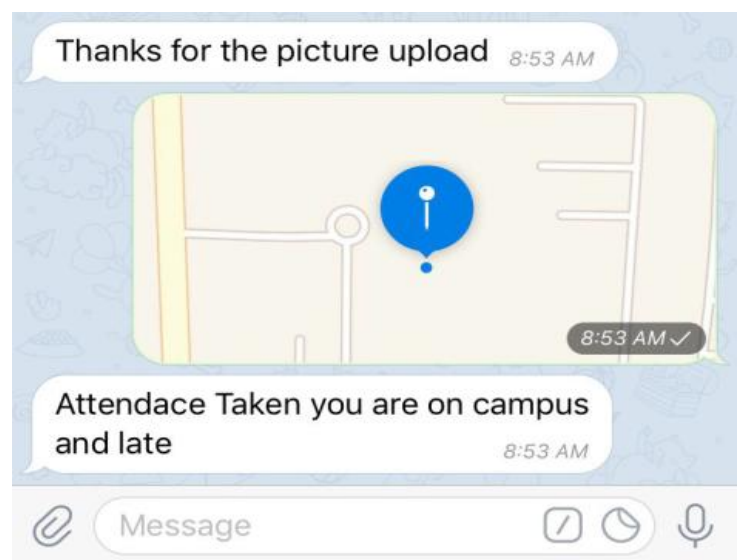

Fig 11: Attendance Recording on iPhone

The table below shows details of the staff whose registration was done via the bot on the Telegram's mobile application. The information contains the picture they uploaded, name and staff identification number together with generated chatid from Telegram. The data shown is from Redis datastore. This table is displayed on the web application with the Thymeleaf template renderer.

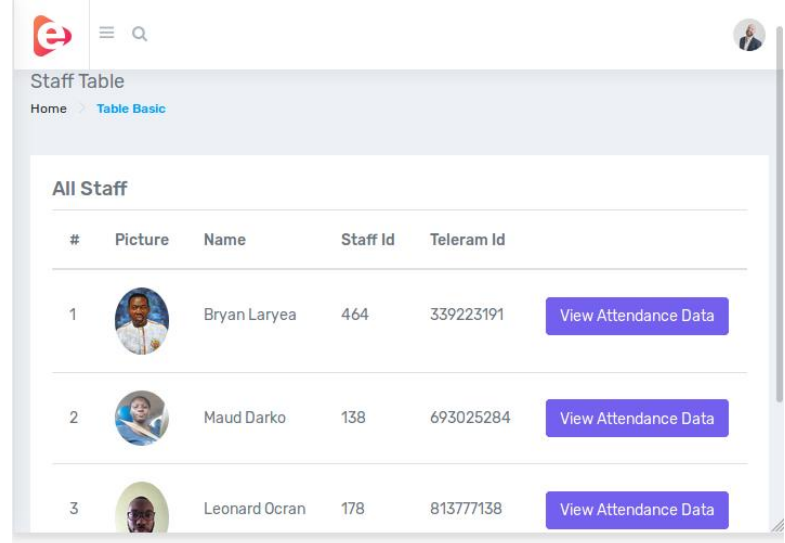

Fig 12: Table Showing Staff.

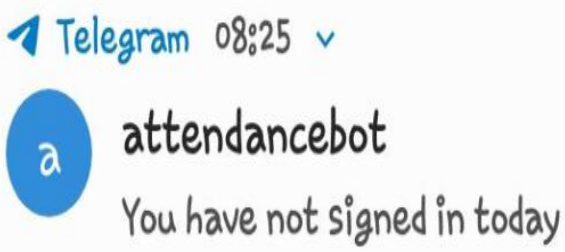

Fig12. Bot Reminder 
Figure 12 shows the android notification from the attendance bot. It also shows our 8:25am attendance reminder scheduler works.

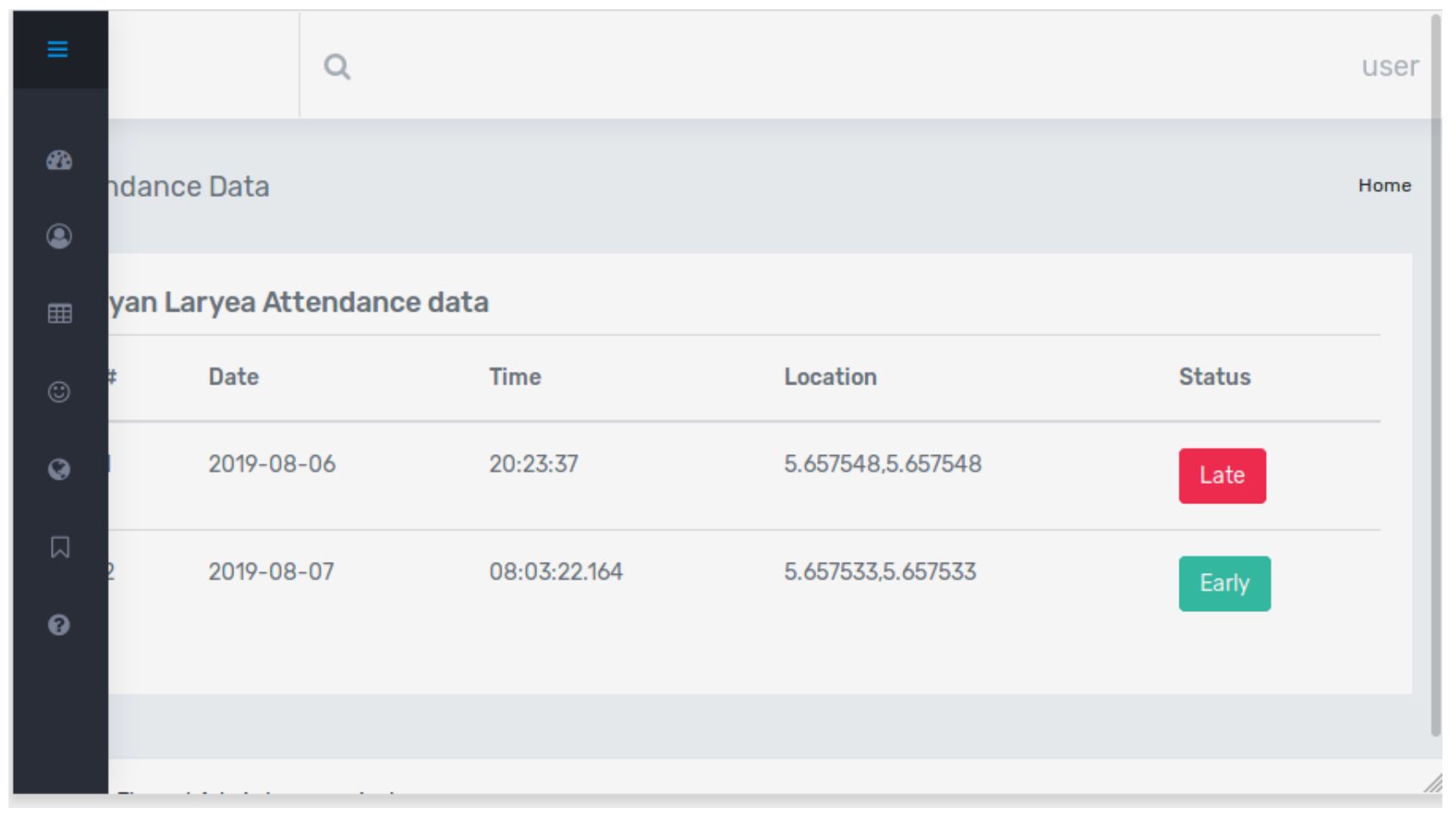

Fig 13: Attendance data of a staff in the web app

A worker's attendance data is shown in Fig. 13. CSS was used to indicate the status of the attendance. Red button for late, Green button for early and blue button for on time as shown.

\section{LIMITATIONS AND RECOMMENDATIONS}

One of the limitations of this research is battery life which is the bane of smartphones. When the worker arrives at the work place with the smartphone off he or she cannot register their attendance. Also, the system requires constant internet both the user and the server housing the bot. In case the company fails to renew the Internet subscription, the server would be off. Similarly, when there is power outage which is not unusual in Ghana, the users would not see the bot online and their confirmation would be delayed. Likewise, if the smartphone user Internet bundle is exhausted they cannot access the bot. One recommendation is deployment of the application on a Virtual Private Server cloud service to ensure 24 hours bot availability. Also, further research should be done on how to create a hybrid between the biometric and this project to make them complementary by addressing each technology demerits.

\section{CONCLUSION}

The application enables users to record their attendance within $250 \mathrm{~m}$ of the device without walking to the device. The time period between location upload to receiving of confirmation was maximum of 2 seconds with the slowest Internet connection. We realized this can be used by the C.S.I.R. Institutes which have offices located all over the country. Each campus can have Geo-Fence instead of purchasing many biometric attendance trackers.

\section{ACKNOWLEDGMENTS}

Our thanks to Dr. William Oduro, Maud Shirley Darko, Leonard Ocran and Hope Gameli Banini and Prince Odoi for their contributions to this experiment.

\section{REFERENCES}

[1] Md. Shakil \& Rabindra Nath Nandi ,2013. Attendance Management System for Industrial Worker using Finger Print Scanner Global. Journal of Computer Science and Technology Graphics \& Vision Volume 13 Issue 6.

[2] Steve Wright, in Strategic Intelligence Management 2013. Emerging Technologies and the Human Rights Challenge of Rapidly Expanding State Surveillance Capacities Mohammad Dastbaz,

[3] Emily Cronkleton .March 14, 2019, What Is the Average Walking Speed of an Adult?https://www.healthline.com/health/exercisefitness/average-walking-speed

[4] Shermin Sultana1, Asma Enayet1 and Ishrat Jahan Mouri. 2015, A Smart, location Based Time and Attendance Tracking System Using Android Application nternational Journal of Computer Science, Engineering and Information Technology (IJCSEIT), Vol. 5,

[5] Ian Ho, Yi-Jen \& Dewan, Sanjeev \& Ho, Yi-Chun. (2018). Distance and Local Competition in Mobile Geofencing..

[6] García-Martín, Julio Manuel \& Alberto Buendía, José. (2019). Polluti-Bot: An informative bot of pollution in Madrid

[7] M.A. Ansari, Shubham Sharma, Ajay Shete, Shirish Ghorpade, Nikhil Ghodke 2017, Event Organization 
Using GPS Based Location Tracking Including Communication System,Imperial Journal of Interdisciplinary Research (IJIR) Vol-3, Issue-6,

[8] Ankur Chandra ,Shashank Jain, Mohammed Abdul Qadeer 2011,GPS Locator: An Application for Location Tracking and Sharing Using GPS for Java Enabled Handhelds International Conference on Computational Intelligence and Communication Networks

[9] Telegrambots Spring Boot starter, https://github.com/rubenlagus/TelegramBots/tree/master/ telegrambots-spring-boot-starter

[10] Geo-fence,https://en.wikipedia.org/wiki/Geo-fence

[11] Patil, P., Khachane, A and Purohit, V. 2016. A Wireless Fingerprint Attendance System. International Journal of Security, Privacy and Trust Management. Vol 5, Issue 4

[12] Redis, https://redis.io/

[13] Stephen Coetzee \& Astrid Schmulian, "Check In" For Class with A Messenger Chatbot, https://chatbotsmagazine.com/check-in-for-class-with-amessenger-chatbot-7089491be7ca

[14] Spatial4J,https://locationtech.github.io/spatial4j/

[15] https://en.wikipedia.org/wiki/Spring_Framework

[16] Sending message to a telegram channel the easy way https://medium.com/@xabaras/sending-a-message-to-aTelegram-channel-the-easy-way-eb0a0b32968\#6ace

[17] Machine Learning Biometric Attendance System using Fingerprint Fuzzy Vault Scheme Algorithm and MultiTask Convolution Neural Network Face Recognition Algorithm, International Journal of Computer Applications.

[18] Li, Jianpo \& Xu-ning, Zhu \& Xue, Li \& Zhi-ming, Zhang \& Sui, Ji-sheng. (2010). Wireless Fingerprint Attendance System Based on ZigBee Technology. 10.1109/IWISA.2010.5473360. 